\title{
Hedgehog signaling is critical for maintenance of the adult coronary vasculature in mice
}

\author{
Kory J. Lavine, ${ }^{1}$ Attila Kovacs, ${ }^{1,2}$ and David M. Ornitz ${ }^{1}$ \\ ${ }^{1}$ Department of Developmental Biology and 'Department of Internal Medicine, Washington University School of Medicine, St. Louis, Missouri, USA.
}

\begin{abstract}
Hedgehog (HH) signaling has emerged as a critical pathway involved in the pathogenesis of a variety of tumors. As a result, $\mathrm{HH}$ antagonists are currently being evaluated as potential anticancer therapeutics. Conversely, activation of $\mathrm{HH}$ signaling in the adult heart may be beneficial, as $\mathrm{HH}$ agonists have been shown to increase coronary vessel density and improve coronary function after myocardial infarction. To investigate a potential homeostatic role for $\mathrm{HH}$ signaling in the adult heart, we ablated endogenous HH signaling in murine myocardial and perivascular smooth muscle cells. HH signaling was required for proangiogenic gene expression and maintenance of the adult coronary vasculature in mice. In the absence of HH signaling, loss of coronary blood vessels led to tissue hypoxia, cardiomyocyte cell death, heart failure, and subsequent lethality. We further showed that $\mathrm{HH}$ signaling specifically controlled the survival of small coronary arteries and capillaries. Together, these data demonstrate that $\mathrm{HH}$ signaling is essential for cardiac function at the level of the coronary vasculature and caution against the use of $\mathrm{HH}$ antagonists in patients with prior or ongoing heart disease.
\end{abstract}

\section{Introduction}

Myocardial infarction (MI) and ischemic heart disease are the leading causes of death in the industrial world. Coronary artery bypass grafting $(\mathrm{CABG})$ and percutaneous coronary interventions (PCIs) are the mainstays of treatment for these patients and significantly reduce morbidity and mortality (1). Unfortunately, nearly $10 \%$ of all patients are ineligible for these procedures for reasons including presence of diffuse or intractable lesions. Additionally, a substantial number of diabetic patients that undergo these procedures show insufficient improvement. Without treatment, many of these patients do poorly, with mortality rates of $8 \%-10 \%$ per year (2).

Recently, a noninvasive approach aimed at promoting growth of new coronary blood vessels has been proposed to treat patients that are not eligible for CABG and PCI. This strategy, termed pharmacological angiogenesis, involves either systemic or local administration of proangiogenic agents, such as FGF or VEGF-A, into the heart. Despite promise in animal models, FGF and VEGF-A therapy has thus far shown little efficacy in clinical studies (2-5).

In search of new candidate pharmacological targets, we and others have discovered that activation of hedgehog $(\mathrm{HH})$ signaling in the adult heart is sufficient to promote coronary neovascularization and protect from ischemia $(6,7)$. These studies directly implicated the $\mathrm{HH}$ signaling pathway as a potential therapeutic target for pharmacological angiogenesis. Interestingly, through investigation of potential effects of $\mathrm{HH}$ activation, it was noted that $\mathrm{HH}$ ligands, most prominently sonic hedgehog $(\mathrm{SHH})$, were expressed in the adult heart (6). Expression of SHH in the adult heart raised the possibility that endogenous $\mathrm{HH}$ signaling may be important for normal cardiac homeostasis or function. Consistent with this, we have previously reported that $\mathrm{HH}$ signaling is essential for the formation of the coronary vascular system during embryonic development (7).

Nonstandard abbreviations used: $\mathrm{HH}$, hedgehog; LAD, left anterior descending artery; MI, myocardial infarction; PTC1, patched1; SHH, sonic hedgehog.

Conflict of interest: The authors have declared that no conflict of interest exists.

Citation for this article: J. Clin. Invest. 118:2404-2414 (2008). doi:10.1172/JCI34561.
To determine whether $\mathrm{HH}$ signaling is required for adult cardiac homeostasis or function, we examined the consequences of acutely removing $\mathrm{HH}$ signaling from the adult mouse heart. We show that temporal and tissue-specific deletion of the essential HH signaling component smoothened ( $\mathrm{Smo}$ ) leads to reductions in proangiogenic gene expression and dropout of coronary blood vessels. Loss of coronary vasculature resulted in tissue hypoxia, cardiomyocyte apoptosis, ventricular failure, and lethality. These data demonstrate that $\mathrm{HH}$ signaling is required in the normal adult mouse heart to maintain proangiogenic gene expression and promote survival of the coronary vasculature.

\section{Results}

SHH and patched 1 are expressed in the adult heart. To identify cell types that express $\mathrm{SHH}$ and potential targets of $\mathrm{HH}$ signaling, we examined expression of $\mathrm{SHH}$ and patched1 (PTC1) in the normal adult mouse heart. Consistent with previous studies, immunohistochemical analysis for $\beta$-galactosidase in Ptc1-LacZ knock-in hearts (8) revealed that PTC1 is expressed in cardiomyocytes and perivascular smooth muscle (Figure 1, A-H). Since PTC1 is both a receptor for and marker of $\mathrm{HH}$ signaling (9), these data suggest that cardiomyocytes and perivascular smooth muscle cells represent 2 potential targets of $\mathrm{HH}$ signaling.

Immunohistochemical examination for SHH protein demonstrated that $\mathrm{SHH}$ was expressed in both the adventitial layer of larger blood vessels and in cells surrounding smaller blood vessels located within the interstitium. Colabeling with Abs against the fibroblast marker vimentin revealed that $\mathrm{SHH}$ was specifically expressed in adventitial and perivascular interstitial fibroblasts (Figure 1, I-L). To gain further insight into the spatial relationship among the coronary microvasculature, $\mathrm{SHH}$-expressing cells, and PTC1-expressing cells, we performed immunohistochemical analysis with Abs against PECAM, SHH, and $\beta$-galactosidase in Ptc1-LacZ knock-in mice (Figure 1, M-P). Colabeling experiments revealed that $\mathrm{SHH}$ and PTC1 were expressed in interstitial cells and cardiomyocytes, respectively, in close association with the coronary microvasculature. 

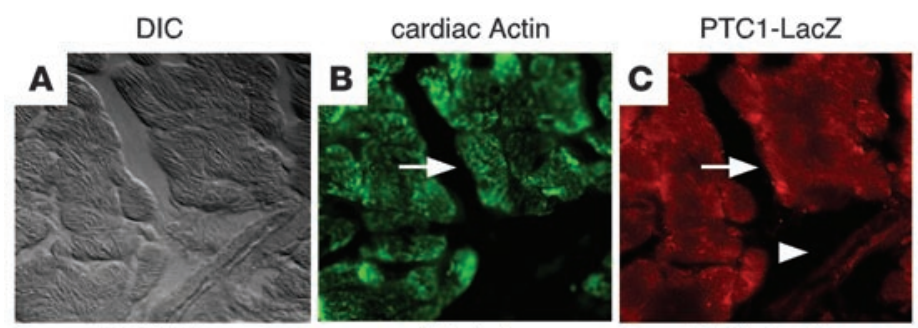

SM Actin
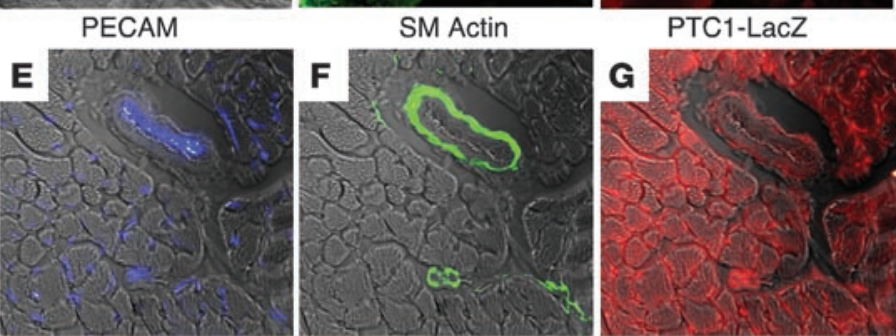

PECAM
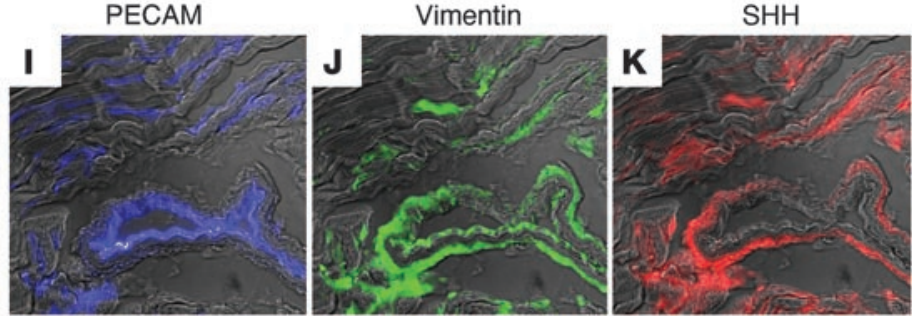

$\mathrm{SHH}$
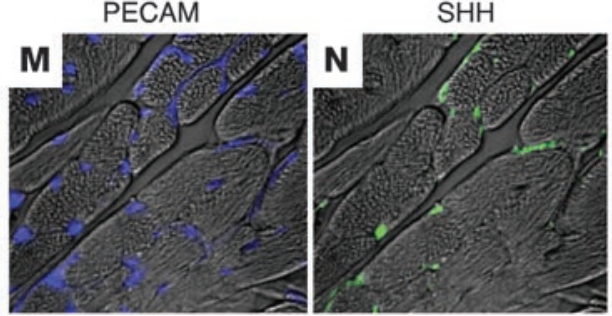

Figure 1

SHH and PTC1 expression in the adult heart. (A-D) Immunohistochemical staining of Ptc1-LacZ adult hearts with Abs against cardiac actin (B, green) and $\beta$-galactosidase $(\mathbf{C}$, red) demonstrating PTC1 expression in cardiomyocytes (white arrows) and large coronary vessels (white arrowheads). A shows the corresponding differential interference contrast (DIC) microscopy image. (E-H) Immunohistochemical staining of Ptc1-LacZ adult hearts with Abs against PECAM (E, blue), SMA $(\mathbf{F}$, green), and $\beta$-galactosidase (G, red). PTC1 expression was detected in both cardiomyocytes and perivascular smooth muscle cells. White arrowheads denote colocalization of PTC1 and SMA expression. (I-L) Immunohistochemical staining with Abs against PECAM (I, blue), vimentin (J, green), and $\mathrm{SHH}$ (K, red) revealed that $\mathrm{SHH}$ is expressed in adventitial and interstitial fibroblasts. White arrowheads denote colocalization of $\mathrm{SHH}$ and vimentin expression. The asterisk indicates area of vimentin and PECAM coexpression. (M-P) Immunostaining with PECAM (M, blue), SHH $(\mathbf{N}$, green), and $\beta$-galactosidase $(\mathbf{O}$, red) demonstrates a close association among $\mathrm{SHH}$-expressing interstitial fibroblasts, PTC1-expressing cardiomyocytes, and blood vessels (white arrowhead). In $\mathbf{E}-\mathbf{G}, \mathbf{I}-\mathbf{K}$, and $\mathbf{M}-\mathbf{O}$, florescent signals are overlaid on top of DIC images. $\mathbf{D}, \mathbf{H}, \mathbf{L}$, and $\mathbf{P}$ show merged images of $\mathbf{A}-\mathbf{C}, \mathbf{E}-\mathbf{G}, \mathbf{I}-\mathbf{K}$, and $\mathbf{M}-\mathbf{O}$, respectively. Original magnification, $\times 400(\mathbf{A}-\mathbf{D}, \mathbf{I}-\mathbf{L}$, and $\mathbf{M}-\mathbf{P}) ; \times 200(\mathbf{E}-\mathbf{H})$.

Together, these data suggest that SHH likely signals to 2 independent populations of cells, perivascular smooth muscle cells and cardiomyocytes. SHH expressed from adventitial fibroblasts may signal to surrounding perivascular smooth muscle cells, potentially affecting larger coronary blood vessel growth or physiology. Additionally, SHH expressed from interstitial fibroblasts may control aspects of coronary microvascular growth or function via signaling to the cardiomyocyte.

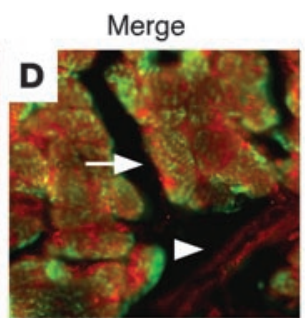

Merge
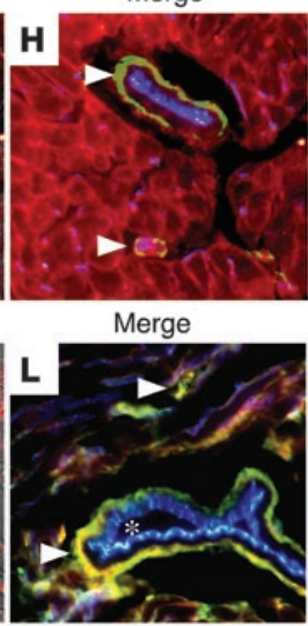

Merge

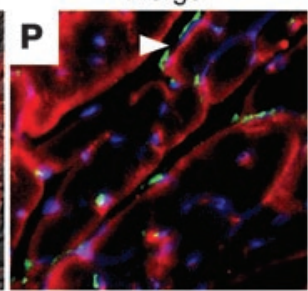

Myocardial HH signaling is required for survival and cardiac function. To identify functions of $\mathrm{HH}$ signaling in the adult heart, we inactivated $\mathrm{HH}$ signaling in the 2 potential targets of $\mathrm{SHH}$, the cardiomyocyte and vascular smooth muscle cell. Myocardiumspecific ablation of $\mathrm{HH}$ signaling in the adult heart was accomplished by conditionally removing a homozygous floxed allele of Smo (10), an essential transducer of $\mathrm{HH}$ signaling, using $\alpha$-myosin heavy chain-ER-Cre ( $\alpha M H C-E R-C r e)$. The $\alpha M H C-E R-C r e$ (mer) transgenic mouse allows spatiotemporal control of Cre recombinase activity in the cardiomyocyte (11). The $\alpha M H C$ promoter drives expression of an ER-Cre fusion protein in the myocardium, and upon administration of tamoxifen, the ERCre protein is translocated into the nucleus, promoting Cre-mediated recombination (Figure 2A). Similarly, the Sm22-Cre mouse was used to target vascular smooth muscle (Figure $2 \mathrm{~B}$ ). Injection of tamoxifen into adult $S m o^{\text {mer }}$ mice resulted in deletion of Smo and ablation of HH signaling in the adult myocardium. Targeting Smo with Sm22-Cre $\left(\mathrm{Smo}^{\mathrm{sm} 22}\right)$ resulted in constitutive ablation of Smo in vascular smooth muscle cells. Mating $S m o^{m e r}$ and $S m o^{s m 22}$ mice to generate $S m o^{\text {mer,sm22 }}$ mice enabled deletion of Smo from both the cardiomyocyte and vascular smooth muscle cell lineages concurrently.

ToremoveHHsignaling from theadult heart, we injected control $\left(S m o^{f l o x / f l o x}\right)$, $S m o^{\text {sm22 }}, S_{m o} o^{\text {mer }}$, and $S m o^{\text {mer,sm22 }}$ mice with tamoxifen once per day for 5 consecutive days (Figure 2C). Treatment with 5 injections of tamoxifen promotes Cre-mediated recombination in up to $90 \%-95 \%$ of cardiomyoctyes (11) (Figure 2A). Following the fifth injection (day 5), Smo ${ }^{\text {mer }}$ and $\mathrm{Smo}^{\text {mer, }, \mathrm{m} 22}$ mice became tachypneic, with ensuing lethality between days 3 and 7 (Figure 2C). $S m o^{\text {mer }}$ and $S m o^{\text {mersm } 22}$ mice that were not injected with tamoxifen were indistinguishable from controls (data not shown). Similarly, mice of the genotype $S m o^{\text {flox1+}}$;mer or $S m o^{\text {floxl+}} ; m e r, S m 22$ that were injected with tamoxifen were also indistinguishable from control mice (data not shown). Unless specifically stated, all further comparisons were made between tamoxifen-injected animals.

Examination of control, $S m o^{s m 22}, S m o^{m e r}$, and $S m o^{m e r, s m 22}$ hearts revealed that by day $5, S m o^{m e r}$ and $S m o^{m e r, s m 22}$ hearts displayed cardiomegaly and ventricular dilation (Figure 2, D-G). Consis- 

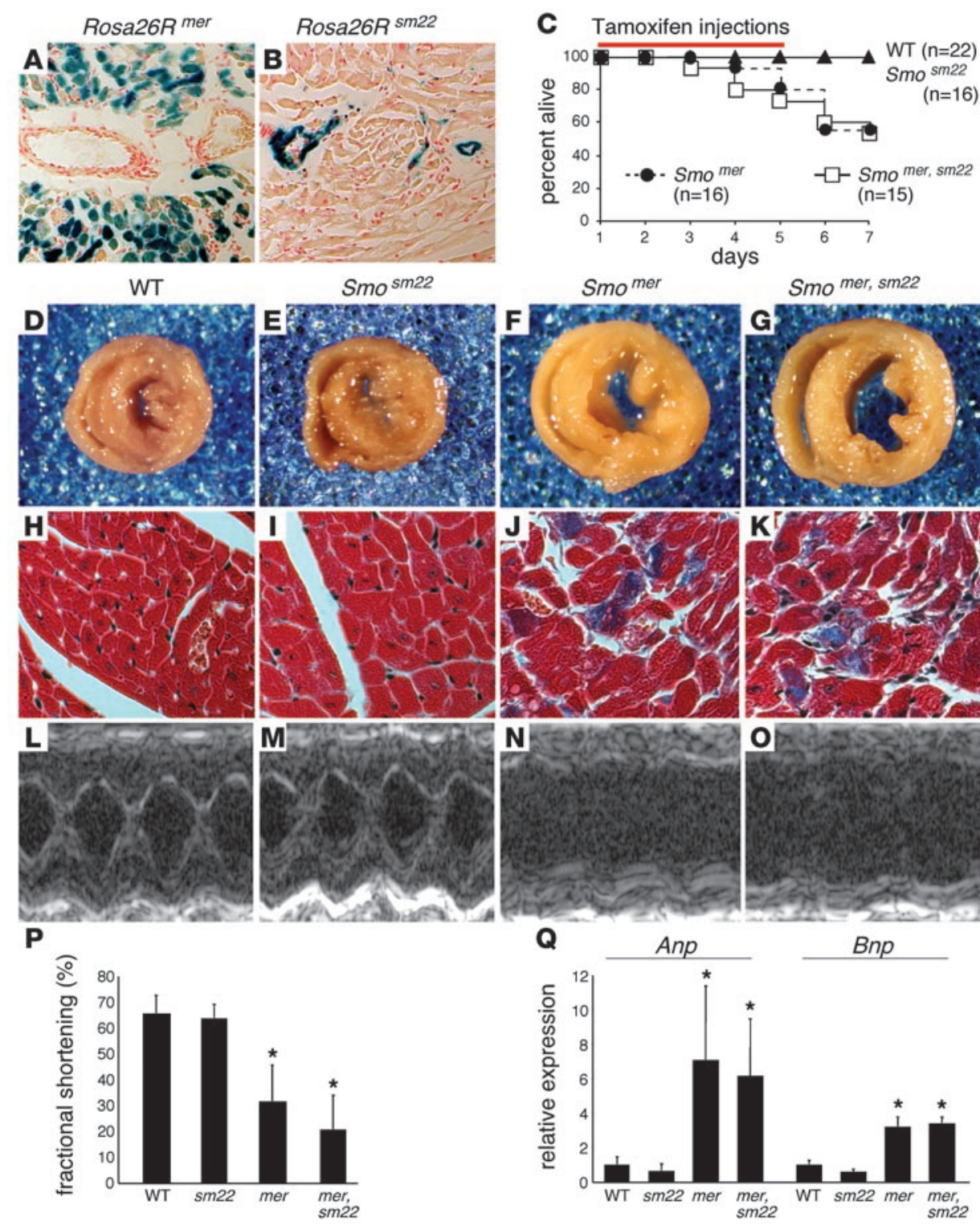

Smo ${ }^{\text {sm22 }}$
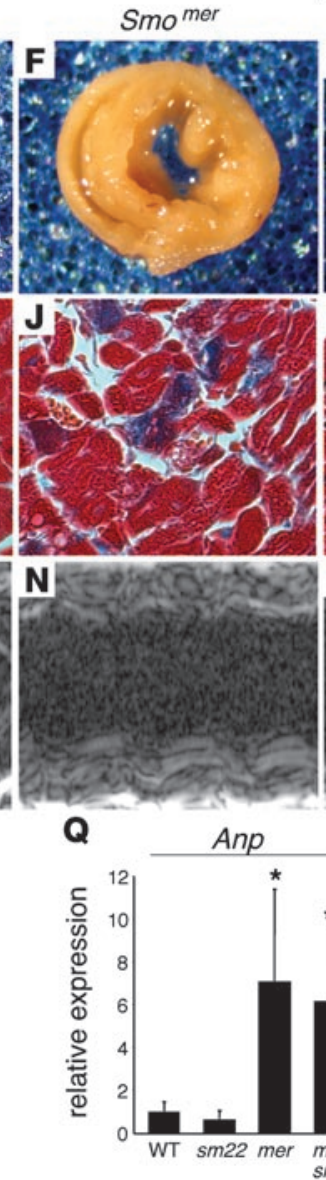

Smo mer, sm22
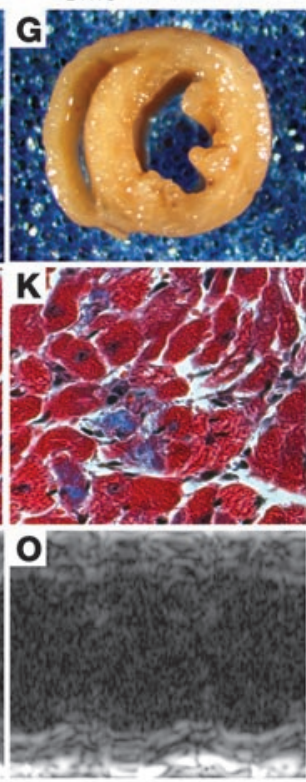

Bnp

\section{Figure 2}

Myocardial $\mathrm{HH}$ signaling is required for survival and cardiac function. (A and $\mathbf{B}) \beta$-Galactosidase staining of

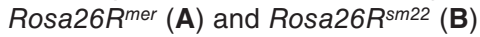
hearts showing Cre-mediated recombination in cardiomyocytes and vascular smooth muscle cells, respectively. Original magnification, $\times 200$. (C) KaplanMeier survival curve demonstrating lethality in Smomer and Smomer,sm22 mice between days 5 and 7 . The red bar denotes the 5-day tamoxifen injection period. (D-G) Whole-mount photographs of transverse heart slices at day 5 , revealing that $S m o^{m e r}(\mathbf{F})$ and $\mathrm{Smo}^{\mathrm{mer}, \mathrm{sm} 22}(\mathbf{G})$ mice have enlarged and dilated ventricles compared with control (D) and Smosm22 (E) mice. Original magnification, $\times 25$. $(\mathbf{H}-\mathbf{K})$ Trichrome staining demonstrating replacement of cardiomyocytes by fibrotic tissue in Smomer $(\mathbf{J})$ and Smomer,sm22 (K) hearts. No fibrosis was seen in control $(\mathbf{H})$ and $\mathrm{Smo}^{\text {sm22 }}$ (I) hearts. Original magnification, $\times 200$. (L-O) 2D M-mode images of control (L), Smosm22 (M), Smomer $(\mathrm{N})$, and $\mathrm{Smo}^{\mathrm{mer}, \mathrm{sm} 22}(\mathrm{O})$ hearts, showing impaired systolic function in Smomer and Smo ${ }^{\text {mer,sm22 }}$ hearts. (P) Quantitation of fractional shortening, demonstrating statistically significant reductions in Smomer and Smomer,sm22 mice compared with control and $\mathrm{Smo}^{\text {sm22 }}$ mice. (Q) Quantitative RT-PCR revealed elevated levels of Anp and Bnp in Smomer and $S m o^{m e r, s m 22}$ hearts compared with control and $S m o^{\text {sm22 }}$ hearts. ${ }^{*} P<0.01$.

tent with cardiac damage and impaired cardiac function, Smo and $S m o^{\text {mersm22 }}$ mice demonstrated fibrotic replacement of myocardial tissue (Figure 2, H-K). Moreover, echocardiographic analysis of fractional shortening in control, $S_{m o} o^{s m 22}, S m o^{m e r}$, and Smo ${ }^{\text {mer, }, m 22}$ mice at day 5 confirmed severely depressed systolic function in $S m o^{\text {mer }}$ and Smo ${ }^{\text {mersm22 }}$ mice (Figure 2, L-P, Table 1, and Supplemental Video 1; available online with this article; doi:10.1172/JCI34561DS1). Serial echocardiography demonstrated persistently diminished systolic function for at least 8 weeks following tamoxifen injections in surviving $S m o^{m e r}$ and $S m o^{\text {mer,sm } 22}$ mice (data not shown). Quantitative RT-PCR revealed elevated levels of Anp and Bnp compared with those in control and $S m o^{\text {sm22 }}$ hearts (Figure $2 \mathrm{Q}$ ).

Cardiomegaly, chamber dilation, impaired systolic function, and fibrotic replacement of myocardial tissue strongly suggest that lethality seen in $S m o^{m e r}$ and $S m o^{m e r, s m 22}$ mice is a result of heart failure. Consistent with this, $S m o^{\text {mer }}$ and $S m o^{\text {mersm22 }}$ mice became tachypneic and demonstrated histological evidence of pulmonary venous congestion and edema (data not shown). These data indicate that cardiac HH signaling is essential for preservation of cardiac function and that acute loss of myocardial $\mathrm{HH}$ signaling leads to heart failure and subsequent lethality.

Myocardial HH signaling is necessary for maintenance of the coronary vasculature. Given the close proximity of $\mathrm{SHH}$-expressing interstitial fibroblasts and PTC1-expressing cardiomyocytes to the coronary microvasculature and the severe cardiac dysfunction seen in injected $S m o^{m e r}$ and $S m o^{m e r, s m 22}$ mice, we hypothesized that myocardial HH signaling may preserve cardiac function by maintaining the coronary vasculature. To test this hypothesis, we examined the coronary vasculature of control, $\mathrm{Smo}^{\mathrm{sm} 22}$, Smo ${ }^{\mathrm{mer}}$, and $S m o^{\text {mersm }} 22$ hearts. PECAM staining after 5 days of tamoxifen injection revealed that $S m o^{\text {mer }}$ and $S m o^{\text {mer,sm22 }}$ hearts contained significantly $(P<0.01)$ fewer numbers of blood vessels $(75.3$ and 64.1 vessels $/ \times 100$ field) compared with $S m o^{\text {sm22 }}$ and control hearts (128 and 137 vessels $/ \times 100$ field) (Figure $3, A-D$, and I). There was a small but statistically significant reduction in blood vessel num- 


\section{Table 1}

Echocardiographic parameters at day 5 of tamoxifen injection

\begin{tabular}{|c|c|c|c|c|c|c|c|c|}
\hline Genotype & LVIDd & LVIDs & LVPWs & LVAWs & LVPWd & LVAWd & $\% \mathrm{FS}$ & RWT \\
\hline Control & $3.1 \pm 0.2$ & $1.0 \pm 0.1$ & $1.4 \pm 0.2$ & $1.6 \pm 0.1$ & $0.6 \pm 0.07$ & $0.6 \pm 0.07$ & $67 \pm 2.4$ & $0.38 \pm 0.06$ \\
\hline$S m o^{\text {sm22 }}$ & $3.2 \pm 0.1$ & $1.1 \pm 0.2$ & $1.3 \pm 0.1$ & $1.6 \pm 0.2$ & $0.6 \pm 0.02$ & $0.7 \pm 0.08$ & $66 \pm 4.6$ & $0.44 \pm 0.03$ \\
\hline Smomer & $4.1 \pm 0.4^{A}$ & $2.7 \pm 0.7^{A}$ & $1.0 \pm 0.2^{A}$ & $1.2 \pm 0.3^{\mathrm{A}}$ & $0.6 \pm 0.09$ & $0.7 \pm 0.11$ & $33 \pm 9.4^{A}$ & $0.32 \pm 0.06$ \\
\hline Smomer,sm22 & $4.3 \pm 0.8^{A}$ & $3.5 \pm 1.1^{\mathrm{A}}$ & $0.8 \pm 0.5^{\mathrm{A}}$ & $1.0 \pm 0.2^{\mathrm{A}}$ & $0.6 \pm 0.10$ & $0.7 \pm 0.07$ & $22 \pm 10^{A}$ & $0.34 \pm 0.02^{A}$ \\
\hline
\end{tabular}

LVIDd, LV internal dimension, diastolic; LVIDs, LV internal dimension, systolic; LVPWs, LV posterior wall thickness, systolic; LVAWs, LV anterior wall thickness, systolic; LVPWd, LV posterior wall thickness, diastolic; LVAWd, LV anterior wall thickness, diastolic; \% FS, percent fractional shortening; RWT, relative wall thickness. ${ }^{A} P<0.05$ compared with control.

ber between $S m o^{\text {mersm22 }}$ and $S m o^{\text {mer }}$ hearts $(P<0.01)$. Labeling with SMA Abs demonstrated that $S m o^{\text {mer }}$ hearts had increased numbers of smooth muscle-positive coronary vessels ( 27.9 vessels $/ \times 20$

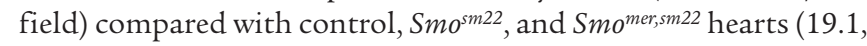
19.1, and 16.4 vessels $/ \times 20$ field; $P<0.01$ ) (Figure 3, E, F, G, H, and J). Smomer,sm22 hearts had a small but statistically significant decrease in smooth muscle cell-containing coronary vessels compared with controls $(P<0.05)$.

These data indicate that $\mathrm{HH}$ signaling to the myocardium is specifically required for maintenance of smaller coronary vessels. In the presence of myocardial $\mathrm{HH}$ signaling, $\mathrm{HH}$ signaling to vascular smooth muscle cells is not necessary for survival of the coronary vasculature. However, in the absence of myocardial $\mathrm{HH}$ signaling, there is an expansion in the number of smooth musclecontaining coronary vessels that is dependent on $\mathrm{HH}$ signaling to perivascular smooth muscle cells.

To further characterize the type of blood vessels affected by loss of cardiac HH signaling, we bred arterial- and venous-specific Efnb2-LacZ and Ephb4-LacZ reporter mice $(12,13)$ into the Smo background. $\beta$-Galactosidase staining of $S m o^{\text {mer,sm22 }} ; E$ fnb2-LacZ and $S m o^{\text {mer,sm22; }}$ Ephb4-LacZ mice revealed that while arterial blood vessels were selectively affected by loss of myocardial $\mathrm{HH}$ signaling, venous blood vessels were spared (Figure 3, K-N).

Consistent with coronary vascular dropout, expression of the proangiogenic factors Vegfa, Vegfb, Vegfc, Ang1, and Ang2 were significantly reduced in $S m o^{\text {mer }}$ and $S m o^{\text {mersm22 }}$ hearts compared with those of controls (Figure 3O). Expression of Vegfd and Ang3 was unaffected (data not shown). Ptc1 expression was similarly diminished in Smo ${ }^{\text {mer }}$ and Smo $\mathrm{Smer}^{\text {, sm22 }}$ hearts, suggesting that the myocardium is the target of the majority of $\mathrm{HH}$ signaling in the adult heart. Together, these data demonstrate that myocardial, and to a much lesser extent vascular smooth muscle, $\mathrm{HH}$ signaling is required for expression of Vegfa, Vegfb, Vegfc, Ang1, and Ang2 and for the maintenance of smaller arterial coronary blood vessels.

Removal of $\mathrm{HH}$ signaling from adult hearts results in cell death and tissue bypoxia. To investigate whether coronary vessel dropout in Smomer and Smomer,sm22 hearts results in cell death and/or tissue hypoxia, we examined histological sections of day 5 control, $S m o^{s m 22}, S m o^{m e r}$, and $S m o^{m e r, s m 22}$ hearts. TUNEL staining revealed

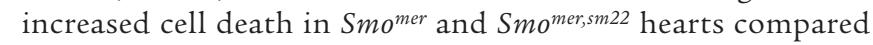
with $S m o^{s m 22}$ and control hearts (Figure 4, A-D). TUNEL staining was present in both cardiomyocytes and coronary endothelial cells, demonstrating that cardiomyocyte cell death and coronary vessel dropout are due to apoptosis. To confirm these results, control, $\mathrm{Smo}^{\mathrm{sm} 22}, \mathrm{Smo}^{\mathrm{mer}}$, and $\mathrm{Smo} \mathrm{o}^{\mathrm{mer}, \mathrm{sm} 22}$ hearts were subjected to active caspase-3 staining. Similar to what was observed with TUNEL staining, Smomer and $S m o^{\text {mer,sm22 }}$ hearts contained increased numbers of active caspase-3-positive cardiomyocytes and coronary endothelial cells compared with $S m o^{\text {sm22 }}$ and control hearts (Figure 4, E-H).

Concurrent coronary blood vessel dropout and cardiomyocyte apoptosis are consistent with the possibility that tissue hypoxia may be the result of lost coronary vasculature and the cause of cardiomyocyte cell death. To determine whether this might be the case, control, $S m o^{s m 22}, S m o^{m e r}$, and $S m o^{m e r, s m 22}$ hearts were directly examined for evidence of tissue hypoxia. Hypoxia was detected using the hypoxyprobe- 1 system (14), which identifies cells that are exposed to a $\mathrm{pO}_{2}$ of less than $10 \mathrm{mmHg}$. Hypoxyprobe- 1 staining demonstrated substantial numbers of hypoxic cardiomyocytes in $\mathrm{Smo}^{\text {mer }}$ and $\mathrm{Smo}^{\text {mer,sm22 }}$ hearts, compared with an absence of hypoxic

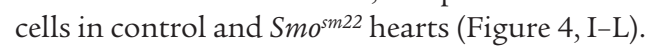

To further investigate whether ventricular failure and cardiomyocyte cell death are a consequence of disrupting coronary vascular density in $S m o^{\text {mer,sm22 }}$ hearts, we temporally defined the sequence by which these events occur. Cardiac dysfunction, as assessed by ventricular dilation, was first present by the fourth day of tamoxifen treatment (Figure 5, A-D). Beginning the third day of tamoxifen treatment, preceding the development of ventricular dilation, a statistically significant reduction in coronary vessel density was evident compared with that in uninjected Smomer,sm22 hearts. Coronary vessel density sharply declined following the fourth and fifth day of tamoxifen treatment (Figure 5 , E-J). Tissue hypoxia was detected beginning on the fourth day of tamoxifen treatment, coinciding with the onset of ventricular dysfunction and significant loss of coronary vessel density (Figure 5, K-N). Cardiomyocyte cell death and fibrotic replacement of cardiac tissue were only detected following the fifth tamoxifen injection (Figure 5, O-V). Together, these data indicate that coronary vascular disruption and subsequent tissue hypoxia, respectively, precede and coincide with the onset of ventricular dysfunction. Moreover, cardiomyocyte cell death and fibrosis occur only following these events, suggesting that loss of coronary vessel density and resultant tissue hypoxia are responsible for ventricular dysfunction, cardiomyocyte apoptosis, and fibrosis in hearts deficient in $\mathrm{HH}$ signaling.

HH signaling is not necessary for survival of cardiomyocytes. To formally exclude the possibility that $\mathrm{HH}$ signaling to cardiomyocytes is directly required for their survival, we investigated whether inhibition of $\mathrm{HH}$ signaling promotes neonatal mouse cardiomyocyte cell death in vitro. Treatment of mouse neonatal cardiomyocytes with cyclopamine did not result in increased cell death compared with vehicle control treatment. In contrast, hydrogen peroxide led to significant increases in cardiomyocyte cell death (Figure 6, A-D). To determine whether sufficient inhi- 

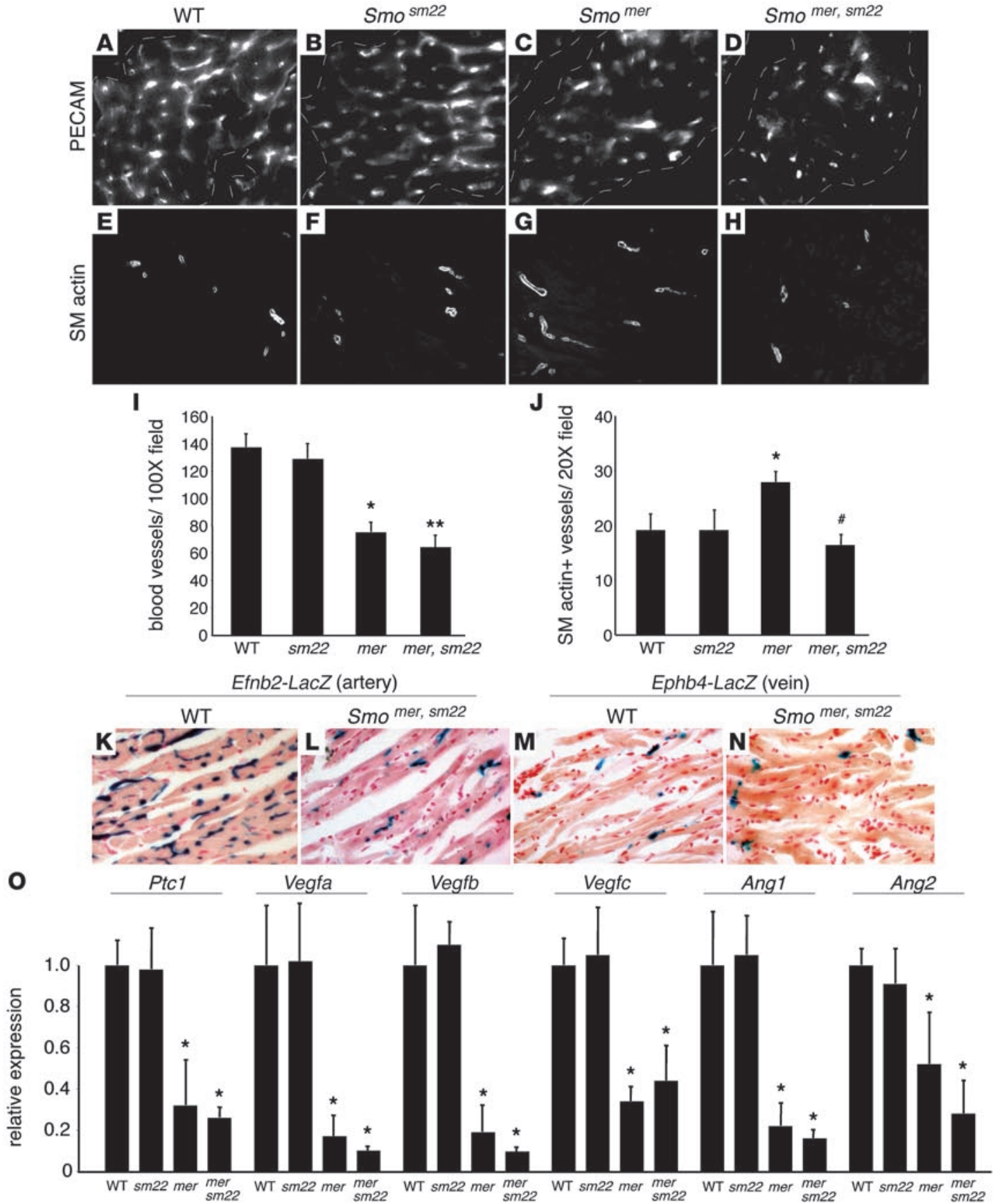

Figure 3

Cardiac $\mathrm{HH}$ signaling is necessary for maintenance of the coronary vasculature. (A-H) Immunohistochemical staining of control (A and E), Smo ${ }^{\text {sm22 }}$ (B and F), Smo ${ }^{m e r}(\mathbf{C}$ and $\mathbf{G})$, and Smo ${ }^{m e r, s m 22}$ (D and $\mathbf{H}$ ) hearts with Abs against PECAM (A-D) and SMA (E-H), demonstrating alterations in blood vessel number and composition in Smomer and Smomersm22 hearts. PECAM staining reveals decreased numbers of total blood vessels in Smomer and $S m o^{m e r, s m 22}$ hearts compared with control and Smosm22 hearts. SMA staining shows increased numbers of smooth muscle-positive blood vessels in $\mathrm{Smo}^{\mathrm{mer}}$ hearts, which is abolished in $\mathrm{Smo} \mathrm{mer}^{\mathrm{m}} \mathrm{m} 22$ hearts. The dashed lines demarcate the boundary of myocardial tissue. Original magnification, $\times 400$ (A-D); $\times 100$ (E-H). (I) Quantitation of the total number of blood vessels/x100 field in control, Smosm22, Smomer, and Smomer,sm22 hearts, revealing a statistically significant reduction in Smomer and $S m o^{m e r, s m 22}$ hearts. Smo ${ }^{m e r, s m 22}$ hearts have a further reduction in blood vessels compared with $S m o^{m e r}$ hearts. ${ }^{\star} P<0.01$ compared with control; ${ }^{\star \star} P<0.01$ compared with control and Smomer hearts. (J) Quantitation of smooth muscle cell-positive blood vessels, showing a statistically significant increase in the number of smooth muscle-positive blood vessels in Smomer hearts ( $\left.{ }^{*} P<0.01\right)$. Smomer,sm22 hearts have a slight reduction in smooth muscle-containing blood vessels compared with controls $(\# P<0.05)$. (K-N) $\beta$-Galactosidase staining for Efnb2-LacZ (artery marker) in control (K) and Smomer,sm22 (L) hearts, demonstrating a reduction in ephrinB2-positive cells in Smomer,sm22 hearts. $\beta$-Galactosidase staining for Ephb4-LacZ (vein marker) in control (M) and Smomer,sm22 (N) hearts, revealing that control and Smomer,sm22 hearts contain similar numbers of ephB4-positive cells. Original magnification, $\times 200$. (0) Quantitative RT-PCR showing diminished Ptc1, Vegfa, Vegfb, Vegfc, Ang1, and Ang2 expression in Smo ${ }^{m e r}$ and Smo ${ }^{m e r, s m 22}$ hearts compared with control and Smosm22 hearts. ${ }^{*} P<0.01$ compared with controls. 


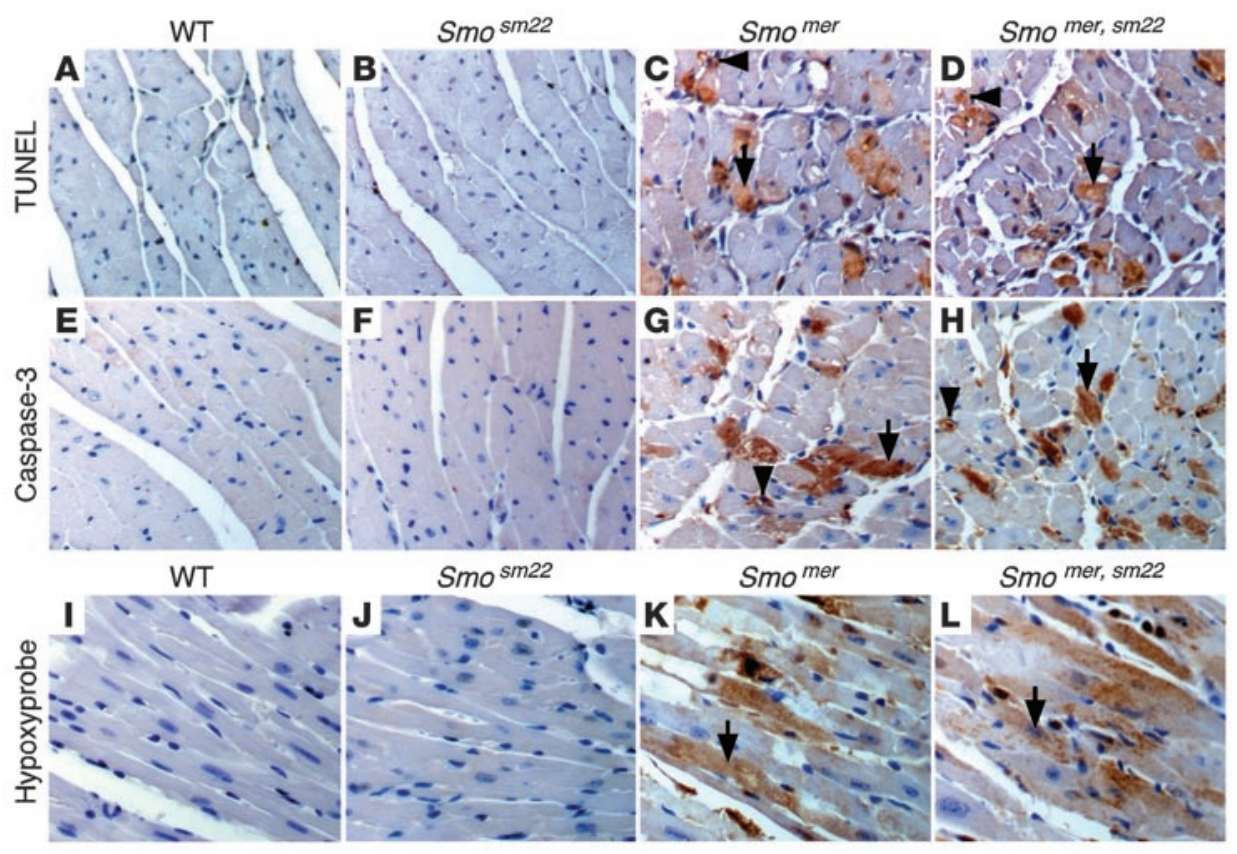

\begin{abstract}
Figure 4
Loss of cardiac $\mathrm{HH}$ signaling results in cell death and tissue hypoxia. (A-D) TUNEL staining revealed increased apoptosis in Smo ${ }^{m e r}$ (C) and $\mathrm{Smo}^{\mathrm{mer}, \mathrm{sm} 22}$ (D) hearts compared with control (A) and Smo ${ }^{\text {sm22 }}$ (B) hearts. TUNEL-positive cardiomyocytes (black arrows) and coronary endothelial cells (black arrowheads) were observed. (E-H) Immunostaining of control (E), Smo ${ }^{\text {sm22 }}$ $(\mathbf{F}), \operatorname{Smo}{ }^{m e r}(\mathbf{G})$, and Smomer,sm22 $(\mathbf{H})$ hearts with Abs against active caspase-3, demonstrating expression in cardiomyocytes (black arrows) and coronary endothelial cells (black arrowheads) in Smomer and Smomer,sm22 hearts. (I-L) Hypoxyprobe-1 staining of control (I), Smo ${ }^{\text {sm22 }}(\mathbf{J}), S^{\text {Smer }}(\mathbf{K})$, and $\mathrm{Smo}^{\mathrm{mer}, \mathrm{sm} 22}(\mathbf{L})$ hearts showing hypoxic cardiomyocytes (black arrows) in $\mathrm{Smo}^{\mathrm{mer}}$ and $\mathrm{Smo}^{\mathrm{mer}, \mathrm{sm} 22}$ hearts. Original magnification, $\times 400$.
\end{abstract}

bition of $\mathrm{HH}$ signaling was achieved, we measured the expression of $2 \mathrm{HH}$-responsive transcripts, Ptc1 and Vegfa. Consistent with inhibition of HH signaling, mRNA expression of both Ptc1 and Vegfa was significantly diminished in response to cyclopamine treatment (Figure 6E).

Endogenous $H H$ signaling is critical for recovery from cardiac ischemia. Previous work has demonstrated that SHH expression is increased following cardiac ischemia (6). To determine whether endogenous $\mathrm{HH}$ activity is important for recovery from myocardial ischemia, neutralizing anti-mouse SHH Abs were administered to wild-type mice following MI. Briefly, wild-type mice underwent surgical ligation of the left anterior descending artery (LAD) and were examined by high-resolution echocardiography the next day to measure baseline cardiac function and infarct size. Mice were subsequently randomized to receive either isotype control or neutralizing $\mathrm{SHH}$ $\mathrm{Ab}$ injections i.p. daily for 14 days. Following treatment, mice underwent a second echocardiographic assessment of cardiac function and infarct area.

An initial mortality was observed in both the control and $\mathrm{SHH}$ $\mathrm{Ab}$-treated groups within 1 day following LAD ligation and is an expected outcome of this MI model. However, several of the mice receiving the $\mathrm{SHH} \mathrm{Ab}$ exhibited an additional mortality between days 4 and 7 (Figure 7A). Echocardiographic assessment of cardiac function on day 14 revealed a diminished ejection fraction in mice receiving the $\mathrm{SHH} \mathrm{Ab} \mathrm{compared} \mathrm{with} \mathrm{controls} \mathrm{(Figure} \mathrm{7,} \mathrm{B} \mathrm{and} \mathrm{C),}$ despite similar ejection fractions on day 1 after MI (Figure 7, D and E). Pairwise analysis revealed that mice treated with the $\mathrm{SHH}$ $\mathrm{Ab}$ underwent a significantly greater decline in ejection fraction compared with those in the control group $(-18.6 \%$ versus $-58.5 \%$; $P<0.01$ ) (Figure 7, D-F).

We also assessed the control and SHH Ab-treated groups for changes in infarct area by both histology and echocardiography (Figure 7, G-J). Compared with the control group, mice receiving the $\mathrm{SHH} \mathrm{Ab}$ demonstrated a significant increase in infarct area. Despite a similar infarct size day 1 after MI, pairwise analysis demonstrated an increase in infarct area in $\mathrm{SHH}$ Ab-treated mice compared with control $\mathrm{Ab}$-treated mice (4.5\% versus $31.2 \%$; $P<0.01$ ) (Figure 7, K-M).

Consistent with increased infarct area, $\mathrm{SHH} \mathrm{Ab}$-treated mice had a reduction in border zone coronary vessel density, as measured at either day 4 or day 14 following MI, compared with control mice (day 4, 126 versus 74 vessels/ $\times 100$ field, and day 14,116 versus 81 vessels $/ \times 100$ field; $P<0.01$ ) (Figure $8, \mathrm{~A}-\mathrm{C}$ ). Importantly, $\mathrm{SHH} \mathrm{Ab}$-treated mice had significantly greater amounts of border zone tissue hypoxia and cell death 4 days after MI (Figure 8, D-G). Consistent with reduced $\mathrm{HH}$ signaling, VEGF-A and PTC1 expression was reduced in the border zone of SHH Ab-treated mice compared with controls on day 4 following MI (Figure 8, H-K). Together, these data indicate that endogenous $\mathrm{HH}$ signaling plays a critical role in the response to cardiac ischemia, both preserving cardiac function and minimizing infarct area, possibly by enhancing border zone vasculature and minimizing tissue hypoxia.

\section{Discussion}

Using spatiotemporal specific gene targeting, we have demonstrated that myocardial $\mathrm{HH}$ signaling is required for the maintenance of the coronary microvasculature. Acute ablation of $\mathrm{HH}$ signaling in the adult mouse heart resulted in coronary vessel dropout, tissue hypoxia, cardiomyocyte apoptosis, heart failure, and lethality. Moreover, reduction of $\mathrm{HH}$ signaling following $\mathrm{MI}$ resulted in worsened cardiac function, decreased border zone vasculature, and increased infarct size. These data demonstrate that $\mathrm{HH}$ signaling is essential for maintenance of the coronary vascular system and heart function in both the normal and ischemic heart.

Coronary vessel dropout, tissue hypoxia, and cardiomyocyte

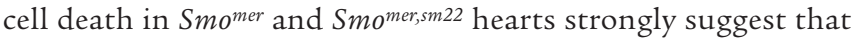
myocardial cell death is due to ischemic injury. These data do not exclude the possibility that myocardial $\mathrm{HH}$ signaling may directly promote cardiomyocyte survival. Treatment of adult cardiomyocytes with $\mathrm{SHH}$ protein in vitro can protect from hydrogen peroxide-induced cell death (6). However, the presence of 
day 0
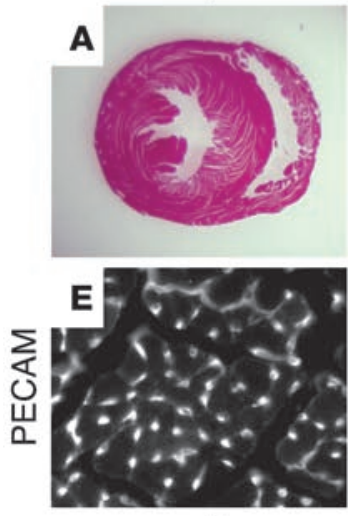

I

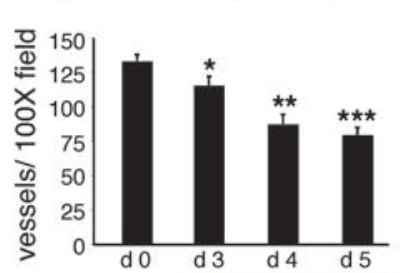

day 3

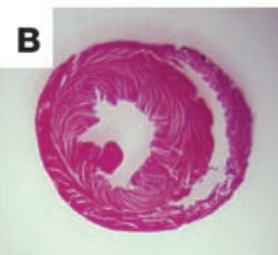

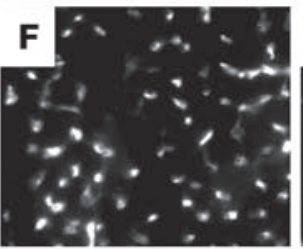
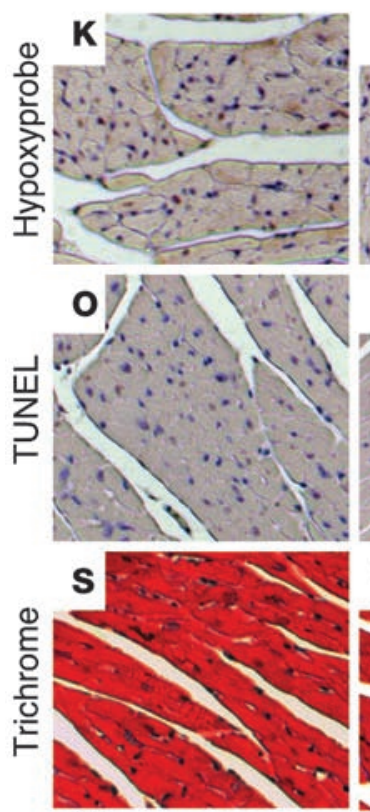

W
$\mathbf{L}$
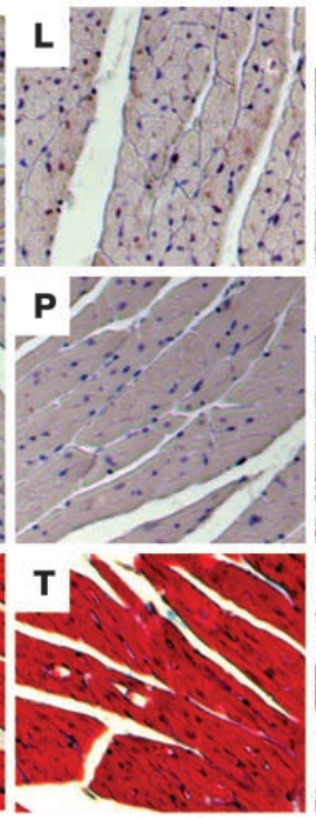

microvascular density day 4
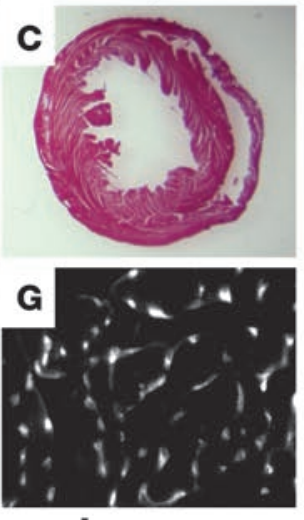

J
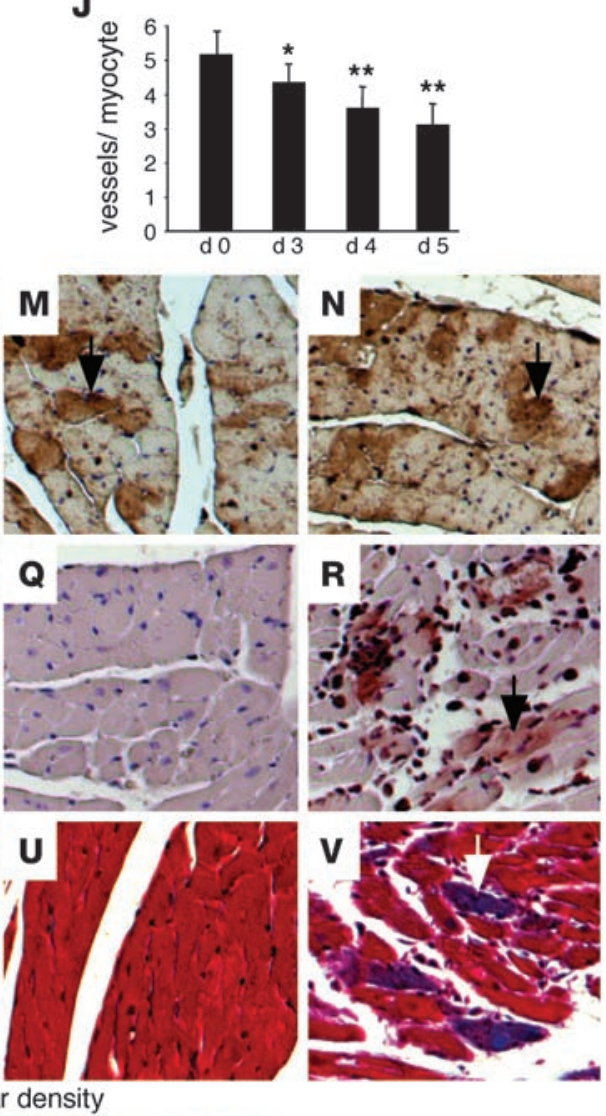

day 5
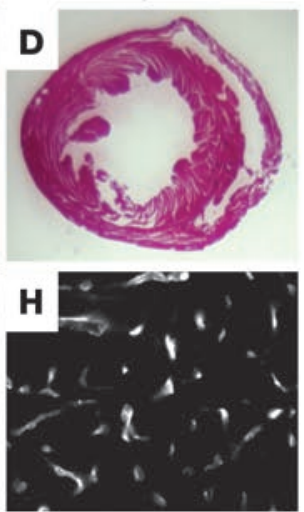

(1)

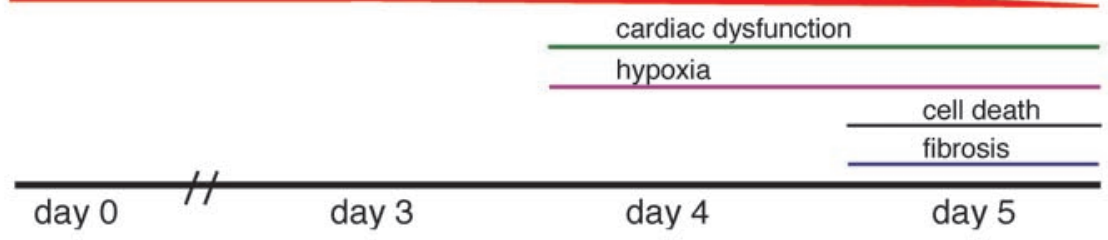

\section{Figure 5}

Coronary blood vessel loss and tissue hypoxia lead to cardiac failure and cardiomyocyte cell death in hearts deficient in $\mathrm{HH}$ signaling. (A-D) H\&E-stained sections of Smomer,sm22 hearts prior to day 0 (A) and after 3 (B), 4 (C), and 5 (D) days of tamoxifen treatment, revealing ventricular dilation beginning at day 4 of tamoxifen treatment. Original magnification, $\times 25$. (E-H) Immunofluorescence staining for PECAM, revealing decreased coronary vessel density beginning after 3 days of tamoxifen treatment (F) compared with baseline (E). Coronary blood vessel density continues to decline after the fourth (G) and fifth $(\mathbf{H})$ days of treatment. Original magnification, $\times 400$. (I and J) Quantification of the number of blood vessels $/ \times 100$ field (I) and the number of blood vessels/myocyte $(\mathbf{J})$, demonstrating continuous decline in vascular density at days 3,4 , and 5 compared with baseline. ${ }^{*} P<0.01$ compared with day 0 ; ${ }^{* \star} P<0.01$ compared with day 3 ; ${ }^{* * \star} P<0.01$ compared with day 4 . (K-N) Hypoxyprobe staining revealing the presence of hypoxic tissue after days 4 and 5 of tamoxifen treatment (black arrows). (O-R) TUNEL staining indicating that cardiomyocyte cell death occurs beginning after day 5 of tamoxifen treatment (black arrow). (S-V) Trichrome staining demonstrating that fibrosis is first detected beginning on the fifth day of tamoxifen treatment (white arrow). Original magnification, $\times 200$. (W) Schematic depicting the sequence of events that occurs following tamoxifen treatment of Smomer,sm22 mice. hypoxic myocardial tissue in $S m o^{\text {mer }}$ and $S m o^{\text {mersm22 }}$ mice indicates that the observed coronary vessel dropout is not only functionally significant but is likely the etiology of cardiomyocyte cell death and ventricular dysfunction.

$H H$ signaling represents a conserved pathway essential for both the embryonic and the adult vasculature. Previously, we have reported that an $\mathrm{HH} / \mathrm{VEGF} /$ angiopoietin pathway controls the forma- tion of the coronary vascular system during development and that reactivation of $\mathrm{HH}$ signaling in the adult heart can promote coronary neovascularization (7). Here, we demonstrate that $\mathrm{HH}$ signaling is not only necessary for coronary vascular development but is required for maintenance of the adult coronary vasculature primarily at the level of the microvasculature. Together, these findings indicate that both the embryonic and adult coro- 


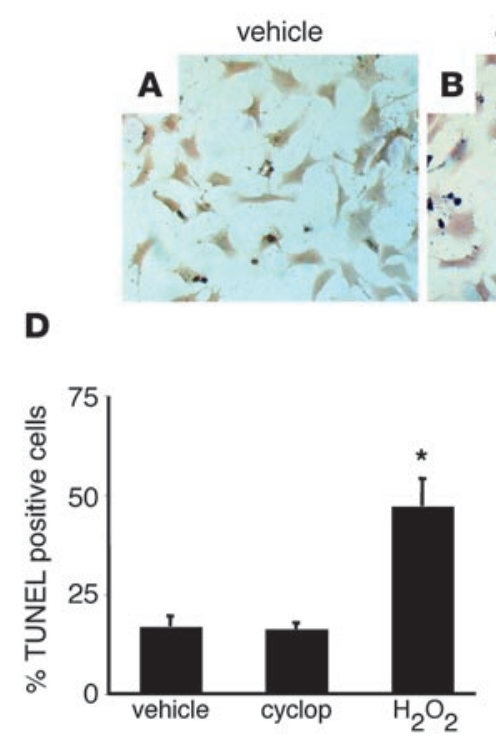

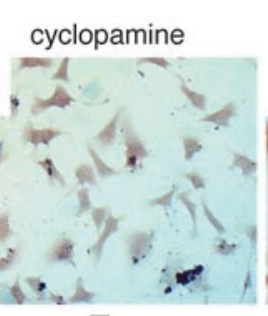



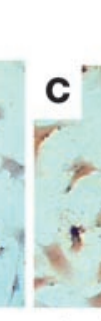

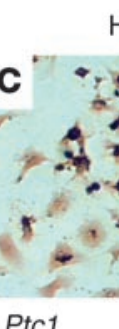
$\mathrm{H}_{2} \mathrm{O}_{2}$

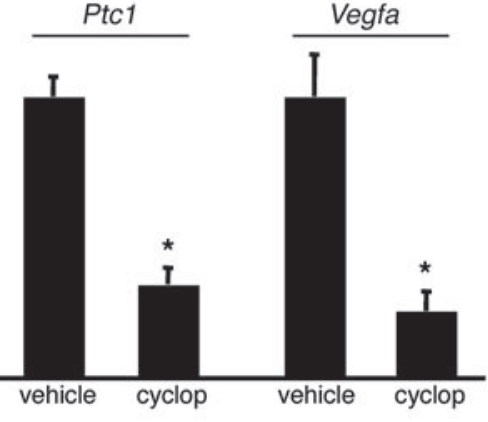

\section{Figure 6}

$\mathrm{HH}$ signaling is not required for survival of mouse neonatal cardiomyocytes. (A-C) TUNEL staining of neonatal mouse cardiomyocytes treated for 24 hours with vehicle (A), $10 \mu \mathrm{M}$ cyclopamine (B), or $0.1 \%$ hydrogen peroxide (C). Original magnification, $\times 200$. (D) Quantification of TUNEL staining, demonstrating that only hydrogen peroxide treatment led to significantly increased cell death compared with vehicle control. cyclop, cyclopamine. (E) Quantitative RT-PCR demonstrating that compared with vehicle control, cyclopamine treatment leads to reduced Ptc1 and Vegfa expression. ${ }^{*} P<0.01$. nary vasculature are regulated by a conserved genetic pathway involving $\mathrm{HH}, \mathrm{VEGF}$, and angiopoietin signaling.

While there is great similarity between embryonic and adult coronary vascular growth, we have noticed some differences. In contrast to embryonic coronary vessel growth, where $\mathrm{HH}$ signaling controls Ang2 expression, in the adult heart, HH signaling regulates both $A n g 1$ and $A n g 2$ expression. Given that ANG1 activates the TIE2 receptor and ANG2 antagonizes TIE2 receptor activation (15), these data suggest that signaling through the TIE2 receptor is differentially required for embryonic coronary vascular growth and adult maintenance. Consistent with this, overexpression of Ang1 in the adult heart inhibits VEGF-induced coronary vessel growth, while overexpression of Ang2 enhances coronary vessel growth (16).

Myocardial HH signaling specifically regulates small coronary arterial vessels. Interestingly, deletion of Smo in the myocardium led to a specific decrease in the coronary arterial but not venous vasculature, suggesting that coronary arteries and veins are supported through distinct mechanisms. Furthermore, hearts deficient in $\mathrm{HH}$ signaling preferentially demonstrated deficits in small vessels including the microvasculature. Whether coronary veins are maintained through a Vegf-dependent mechanism or another system is unclear. However, transgenic mice that specifically express Vegfa in the myocardium demonstrate selective increases in coronary arteries but not veins, indicating that coronary veins might not be a target of VEGF signaling (16).

Determination of whether and which VEGF receptors are expressed in coronary endothelial cells will be necessary to adequately address this question. It is possible that in addition to lymphatic vessels, coronary veins express VEGFR3. This would have interesting ramifications, suggesting potential coregulation of venous and lymphatic growth. Consistent with this, developing veins express VEGFR3 in zebrafish (17). Alternatively, coronary veins could be controlled via VEGF-independent mechanism(s).

Implications for disease and therapeutics. Given that Shb expression is induced following myocardial ischemia and that $\mathrm{HH}$ signaling is necessary and sufficient for coronary vascular growth, it seems likely that $\mathrm{HH}$ signaling constitutes an important mechanism to limit the extent of damage following myocardial ischemia and
MI. This notion is supported by experiments demonstrating that reductions in $\mathrm{HH}$ signaling worsen cardiac function and increase infarct size following MI. These findings not only indicate that $\mathrm{HH}$ signaling plays a critical role following myocardial ischemia, but also suggest that endogenous $\mathrm{HH}$ activity may serve as a biomarker predictive of the extent of recovery after MI. That is, $\mathrm{HH}$ activity may reflect the potential to develop new coronary vasculature in borderline ischemic areas, limiting infarct expansion and further loss of cardiac function. Identification of patients with a low probability of expected recovery will undoubtedly improve the ability to optimally manage and select appropriate interventions after MI. Further studies are warranted to determine whether measurements of $\mathrm{HH}$ ligands or reporters of $\mathrm{HH}$ activity can be used in this way.

In addition to ischemic heart disease, our findings have important implications for the use of $\mathrm{HH}$ antagonists. Currently, $\mathrm{HH}$ antagonists are being developed for the treatment of several cancers, including basal cell carcinoma, pancreatic carcinoma, prostate carcinoma, chondroblastoma, and medulloblastoma (18-25). Given the profound effect of removing $\mathrm{HH}$ signaling from the myocardium, caution should be taken in the development of these pharmaceuticals. It is possible that therapeutic effects of $\mathrm{HH}$ antagonists may be achieved at levels of $\mathrm{HH}$ inhibition that do not impair cardiac function. The dose of the anti-SHH Ab that we administered resulted in no obvious change in cardiac function in control animals (data not shown). However, use of $\mathrm{HH}$ antagonists in patients with prior or ongoing cardiovascular disease may significantly increase the risk of further damage and should only proceed following careful investigation.

\section{Methods}

Tamoxifen treatment. All animal experiments were approved by the Washington University Animal Studies Committee. To inactivate HH signaling in the myocardium, 3- to 4-month-old control (Smo $0^{\text {flox } / f l o x}$ ), Smo ${ }^{s m 22}$, $S m o^{m e r}$, and $S m o^{\text {mersm22 }}$ mice were injected i.p. with $0.1 \mathrm{mg} / \mathrm{g}$ tamoxifen (Sigma-Aldrich) once per day for 5 consecutive days. Tamoxifen was dissolved in sunflower oil (Sigma-Aldrich). All mice were backcrossed at least 3 generations onto the C57BL/6J background. The Rosa26R mouse (26) was used as a reporter for efficiency and localization of ER-Cremediated gene recombination in the presence of tamoxifen. 
A

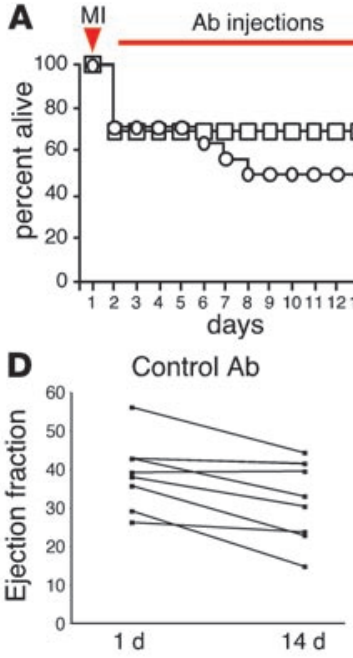

Control Ab (14 d)

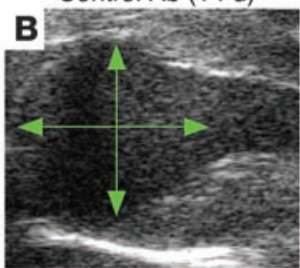

$\mathrm{SHH} A b(14 d)$
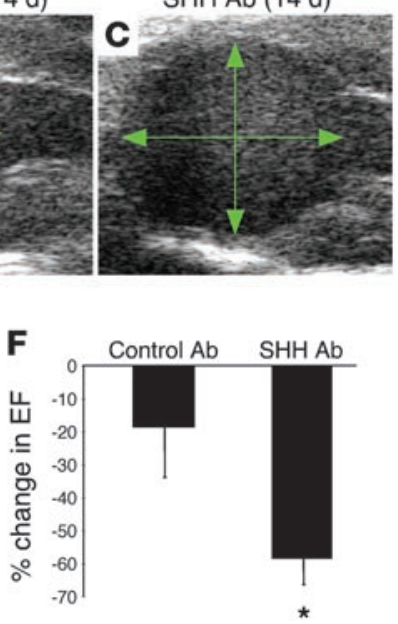

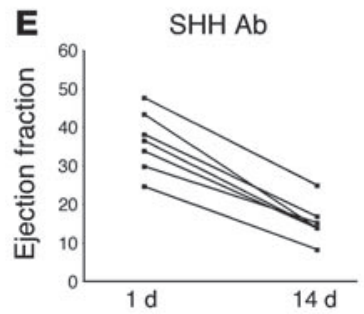

Control Ab (14 d)

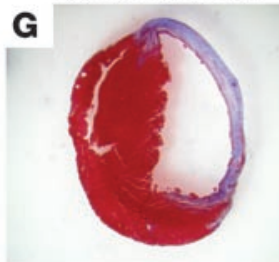

$\mathrm{SHH}$ Ab (14 d) Control Ab (14 d)
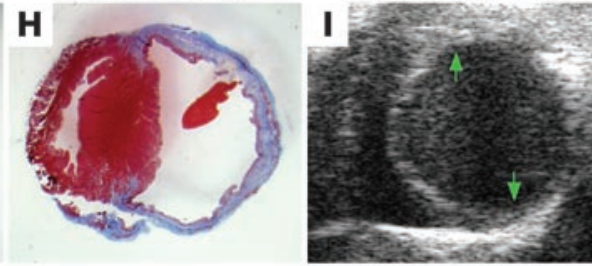

SHH Ab (14 d)
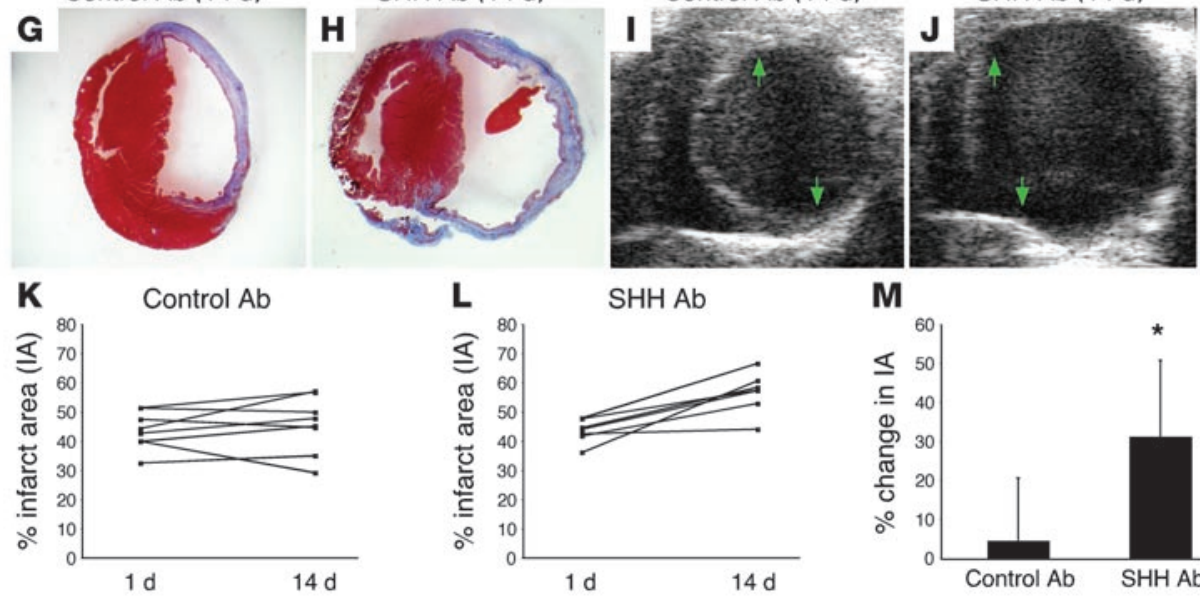

M

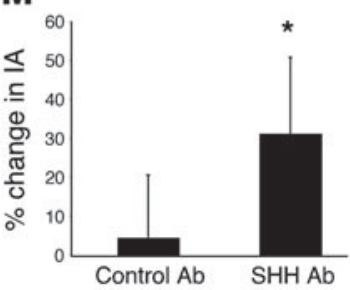

Figure 7

Endogenous $\mathrm{HH}$ signaling is critical for recovery following cardiac ischemia. (A) Kaplan-Meier survival curve showing the initial lethality following LAD ligation in both control and SHH Ab groups. However, between days 4 and 7 after MI, a fraction of the mice receiving SHH Ab died. The red arrow denotes the time at which mice underwent LAD ligation, and the red bar indicates the days mice received Ab injections. (B and $\mathbf{C}$ ) Longaxis end-systolic images of control $(\mathbf{B})$ and $\mathrm{SHH}$ Ab-treated $(\mathbf{C})$ hearts, demonstrating diminished ventricular function in SHH Ab-treated mice. Green arrows highlight the LV cavity. (D and E) Pairwise comparison of ejection fraction 1 day after MI (prior to Ab injections) and 14 days after $\mathrm{MI}$ (after Ab injections) in control (D) and SHH Ab-treated (E) mice. (F) Quantitation of the relative change in ejection fraction, demonstrating that SHH Ab-treated mice undergo a more severe decline in cardiac function compared with control mice $\left({ }^{*} P<0.01\right)$. (G-J) Trichrome staining $(\mathbf{G}$ and $\mathbf{H})$ and short-axis echocardiographic images ( $\mathbf{I}$ and $\mathbf{J})$ of control $(\mathbf{G}$ and $\mathbf{I})$ and $\mathbf{S H H}$ Ab-treated $(\mathbf{H}$ and $\mathbf{J})$ mice showing increased infarct size in SHH Ab-treated animals. Green arrows denote the infarct border zones. Original magnification, $\times 25(\mathbf{G}$ and $\mathbf{H})$. (K and $\mathbf{L}) \mathbf{P a i r w i s e}$ comparison of infarct area 1 day after $\mathrm{Ml}$ (prior to Ab injections) and 14 days after $\mathrm{Ml}$ (after Ab injections) in control (K) and SHH Ab-treated (L) mice. (M) Quantitation of the relative change in infarct area (IA), demonstrating that SHH Ab-treated mice undergo an exaggerated increase in infarct area compared with control mice $\left({ }^{*} P<0.01\right)$.

Histology. Tissues were fixed in $4 \% \mathrm{PFA}$ overnight at $4^{\circ} \mathrm{C}$, dehydrated in $70 \%$ ethyl alcohol, and embedded in paraffin. Four-micrometer sections were cut and stained with either H\&E or trichrome.

Immunohistochemistry and $\beta$-galactosidase staining. For colabeling experiments, tissues were fixed in $2 \%$ PFA overnight at $4{ }^{\circ} \mathrm{C}$, embedded in OCT, infiltrated with sucrose, and frozen, and 10- to $12-\mu \mathrm{m}$ cryosections were cut. Primary Abs used were: PECAM (R\&D), 1:200; SMA (SigmaAldrich), 1:400; LacZ (Abcam), 1:250; vimentin (Chemicon; Millipore), 1:400; and SHH (5E1; Developmental Studies Hybridoma Bank), 1:100. The following secondary Abs were used at 1:200: anti-rat Alexa Fluor 555, anti-rabbit Alexa Fluor 488, anti-rabbit Alexa Fluor 647, anti-goat Alexa Fluor 647, and anti-mouse Alexa Fluor 488 (Molecular Probes).
Immunofluorescence was visualized on a Zeiss ApoTome Microscopy system. Blood vessel number was quantified by examining at least 3 sections from 4 independent samples.

For caspase-3 staining, 4- $\mu \mathrm{m}$ paraffin sections were deparaffinized, rehydrated, peroxidase blocked, and antigen exposed by boiling in $10 \mathrm{mM}$ citric acid. The primary Ab was anti-active caspase-3 Ab (Cell Signaling Technology Inc.), 1:200. The secondary Ab was biotinylated anti-rabbit (Vector Laboratories), 1:200. Signal was detected using the Elite ABC Kit (Vector Laboratories).

TUNEL staining was performed on paraffin-embedded sections as specified by the manufacturer (In Situ Cell Death Detection Kit; R\&D Systems). LacZ staining was performed as described previously (26). 

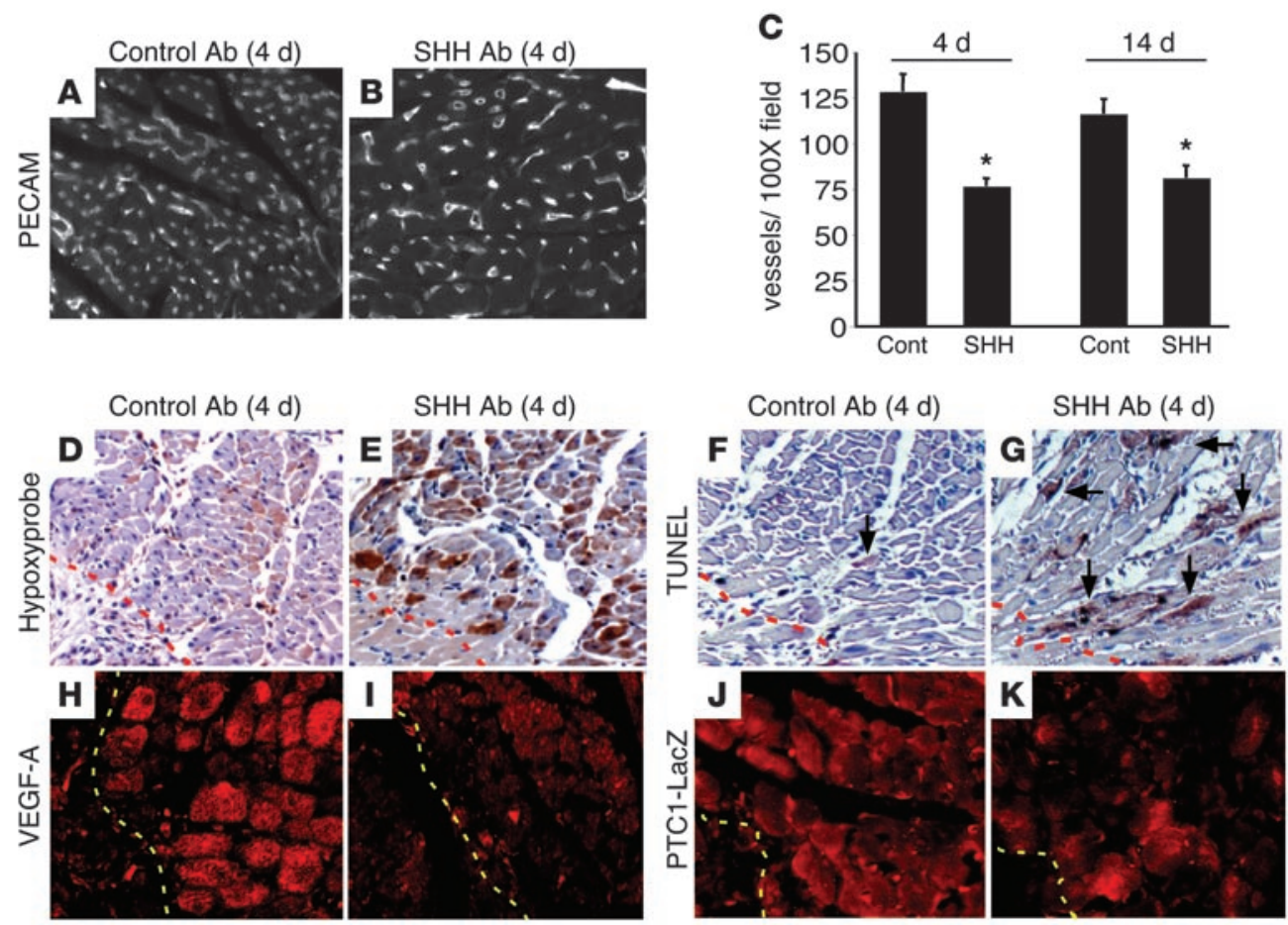

Figure 8

Endogenous $\mathrm{HH}$ signaling is required for survival of the border zone vasculature. (A and B) PECAM staining of the border zone of control (A) and $\mathrm{SHH}$ Ab-treated (B) hearts 4 days after MI, demonstrating that SHH Ab-treated animals have diminished border zone vasculature compared with controls. Original magnification, $\times 100$. (C) Quantitation of blood vessel number $/ \times 100$ field reveals that compared with controls (Cont), SHH $\mathrm{Ab}$-treated hearts contain a statistically significant decrease in the number of blood vessels located within the border zone at days 4 and 14 after $\mathrm{MI}\left({ }^{\star} P<0.01\right)$. (D and $\left.\mathbf{E}\right)$ Hypoxyprobe staining revealing increased hypoxic tissue in the border zone of SHH Ab-treated hearts (E) compared with controls (D). (F and G) TUNEL staining demonstrating that SHH Ab-treated hearts (G) have increased border zone cell death of cardiomyocytes (arrows) compared with controls $(\mathbf{F})$. ( $\mathbf{H}-\mathbf{K})$ Immunofluorescence staining for VEGF-A $(\mathbf{H}$ and $\mathbf{I})$ and $\beta$-galactosidase $(\mathbf{J}$ and $\mathbf{K})$ in control $(\mathbf{H}$ and $\mathbf{J})$ and SHH Ab-treated (I and K) PTC1-LacZ hearts, revealing that SHH Ab treatment leads to reduced VEGF-A and PTC1 border zone expression. Dashed lines indicate the border of the infarct. Original magnification: $\times 200(\mathbf{D}-\mathbf{G}) ; \times 400(\mathbf{H}-\mathbf{K})$.

Hypoxyprobe-1 staining. Mice were injected i.p. with $60 \mathrm{mg} / \mathrm{kg}$ hypoxyprobe-1 (Chemicon; Millipore). Thirty minutes following injection, mice were sacrificed, and hearts were dissected and fixed as specified for routine histological analysis. Four-micrometer paraffin-embedded sections were stained with the anti-hypoxyprobe- $1 \mathrm{Ab}$ as per the manufacturer's instructions.

RNA isolation, cDNA synthesis, and quantitative RT-PCR. RNA was isolated from 4 independent biological samples using the RNeasy kit (QIAGEN). cDNA was obtained using the SuperScript II First-Strand cDNA Synthesis kit (Invitrogen). Quantitative RT-PCR was performed on an ABI 7900 machine using TaqMan probes for Vegfa, Vegfb, Vegfc, Vegfd, Ang1, Ang2, Ang3, and Hprt (ABI; Applied Biosystems). All samples were normalized to Hprt and then scaled relative to controls, where control samples were set at a value of 1 . Thus, results for all experimental samples were graphed as relative expression compared with control. Error bars represent SD. $P$ values were calculated using Student's $t$ test.

Neonatal cardiomyocyte isolation. Mouse neonatal cardiomyocytes were isolated and cultured using the Neonatal Cardiomyocyte Isolation System (Worthington Biochemical Corp.). Cells were plated overnight in serumcontaining medium and then switched to serum-free medium the following morning. Cells were then treated with vehicle, $10 \mu \mathrm{M}$ cyclopamine, or $0.1 \%$ hydrogen peroxide for 24 hours and then assayed for cell death (TUNEL), and RNA was harvested as described above.

Echocardiography. Mouse echocardiography was performed in the Washington University Mouse Cardiovascular Phenotyping Core facility using an ACUSON Sequoia Echocardiography System. High-resolution echocardiography (VisualSonics) was utilized for phenotypic analysis of cardiac function and infarct area for the MI studies (see below).

$M I$. Briefly, mice were randomized to receive either anti-mouse SHH Ab (5E1; Developmental Studies Hybridoma Bank) or isotype control (6B3; Developmental Studies Hybridoma Bank). Prior to Ab injection, mice underwent LAD ligation as previously described (27). On day 1 after MI, mice underwent echocardiography to access infarct area and ejection fraction. Following this analysis, the mice were injected i.p. with either control or SHH Ab daily for 14 days. Mice received $10 \mathrm{mg} / \mathrm{kg}$ on days $1-3$ and $5 \mathrm{mg} / \mathrm{kg}$ thereafter. On day 14 after MI, mice underwent a second echocardiographic assessment and were then sacrificed. Ejection fraction was calculated by measuring the end systolic and diastolic dimensions from long-axis images. Infarct area was calculated by examining serial short-axis images (0.1 mm apart) spanning from the base of the heart (level of aortic valve) to the apex. The percent area of the ventricle that was infarcted was calculated by determining the percent of the ventricle that was akinetic. The percent change in ejection fraction or infarct area was calculated for each mouse. The average percent change was then calculated for the control and SHH Ab groups and compared using standard Student's $t$ test analysis.

Statistics. Data are expressed as mean \pm SD. Student's $t$ test was used for comparisons between groups. $P$ values of less than 0.05 were considered significant. $P$ values of less than 0.01 are additionally identified in the text, figures, and figure legends. 


\section{Acknowledgments}

We thank F. Long for providing Smo mice, J. Molketin for $\alpha M H C$-ER-Cre mice, M. Shipley for Sm22-Cre mice, and D. Anderson for Efnb2-LacZ and Ephb4-LacZ mice. We would also like to thank C. Smith for technical support and the members of the Mouse Cardiovascular Phenotyping Core for their expertise and efforts in physiological assessment of Smo-conditional-KO hearts. This work was funded by NIH grant HL076664, American Heart Association predoctoral fellowship 0415469Z (to K.J. Levine), and a generous contribution from the Virginia Friedhofer Charitable Trust.

Received for publication November 26, 2007, and accepted in revised form May 14, 2008.

Address correspondence to: David M. Ornitz, Washington University School of Medicine, Department of Developmental Biology, Campus Box 8103, 660 S. Euclid Avenue, St. Louis, Missouri 63110, USA. Phone: (314) 362-3908; Fax: (314) 362-7058; E-mail: dornitz@wustl.edu.
1. Caines, A.E., et al. 2004. Outcomes of coronary artery bypass grafting versus percutaneous coronary intervention and medical therapy for multivessel disease with and without left ventricular dysfunction. Cardiology. 101:21-28.

2. Syed, I.S., Sanborn, T.A., and Rosengart, T.K. 2004 Therapeutic angiogenesis: a biologic bypass. Cardiology. 101:131-143.

3. Simons, M., et al. 2002. Pharmacological treatment of coronary artery disease with recombinant fibroblast growth factor-2: double-blind, randomized, controlled clinical trial. Circulation. 105:788-793.

4. Losordo, D.W., et al. 2002. Phase $1 / 2$ placebocontrolled, double-blind, dose-escalating trial of myocardial vascular endothelial growth factor 2 gene transfer by catheter delivery in patients with chronic myocardial ischemia. Circulation. 105:2012-2018.

5. Henry, G.D., et al. 2000. Intracavernosal injections of vascular endothelial growth factor protects endothelial dependent corpora cavernosal smooth muscle relaxation in the hypercholesterolemic rabbit: a preliminary study. Int. J. Impot. Res. 12:334-339.

6. Kusano, K.F., et al. 2005. Sonic hedgehog myocardial gene therapy: tissue repair through transient reconstitution of embryonic signaling. Nat. Med. 11:1197-1204.

7. Lavine, K.J., et al. 2006. Fibroblast growth factor signals regulate a wave of Hedgehog activation that is essential for coronary vascular development. Genes Dev. 20:1651-1661.

8. Goodrich, L.V., Milenkovic, L., Higgins, K.M., and Scott, M.P. 1997. Altered neural cell fates and medulloblastoma in mouse patched mutants. Science. 277:1109-1113.

9. Pearse, R.V., 2nd, Vogan, K.J., and Tabin, C.J. 2001.
Ptc1 and Ptc2 transcripts provide distinct readouts of Hedgehog signaling activity during chick embryogenesis. Dev. Biol. 239:15-29.

10. Long, F., Zhang, X.M., Karp, S., Yang, Y., and McMahon, A.P. 2001. Genetic manipulation of hedgehog signaling in the endochondral skeleton reveals a direct role in the regulation of chondrocyte proliferation. Development. 128:5099-5108.

11. Sohal, D.S., et al. 2001. Temporally regulated and tissue-specific gene manipulations in the adult and embryonic heart using a tamoxifen-inducible Cre protein. Circ. Res. 89:20-25.

12. Gerety, S.S., Wang, H.U., Chen, Z.F., and Anderson, D.J. 1999. Symmetrical mutant phenotypes of the receptor $\mathrm{EphB} 4$ and its specific transmembrane ligand ephrin-B2 in cardiovascular development. Mol. Cell. 4:403-414.

13. Wang, H.U., Chen, Z.F., and Anderson, D.J. 1998. Molecular distinction and angiogenic interaction between embryonic arteries and veins revealed by ephrin-B2 and its receptor Eph-B4. Cell. 93:741-753.

14. Kennedy, A.S., et al. 1997. Proliferation and hypoxia in human squamous cell carcinoma of the cervix: first report of combined immunohistochemical assays. Int. J. Radiat. Oncol. Biol. Phys. 37:897-905.

15. Ward, N.L., and Dumont, D.J. 2002. The angiopoietins and Tie2/Tek: adding to the complexity of cardiovascular development. Semin. Cell Dev. Biol. 13:19-27.

16. Visconti, R.P., Richardson, C.D., and Sato, T.N. 2002. Orchestration of angiogenesis and arteriovenous contribution by angiopoietins and vascular endothelial growth factor (VEGF). Proc. Natl. Acad. Sci. U. S. A. 99:8219-8224.

17. Lawson, N.D., et al. 2001. Notch signaling is required for arterial-venous differentiation during embryonic vascular development. Development.
128:3675-3683

18. Sanchez, P., Clement, V., and Ruiz i Altaba, A. 2005. Therapeutic targeting of the Hedgehog-GLI pathway in prostate cancer. Cancer Res. 65:2990-2992.

19. Williams, J.A. 2003. Hedgehog signaling pathway as a target for therapeutic intervention in basal cell carcinoma. Drug News Perspect. 16:657-662.

20. Williams, J.A., et al. 2003. Identification of a small molecule inhibitor of the hedgehog signaling pathway: effects on basal cell carcinoma-like lesions. Proc. Natl. Acad. Sci. U. S. A. 100:4616-4621.

21. Sanchez, P., and Ruiz i Altaba, A. 2005. In vivo inhibition of endogenous brain tumors through systemic interference of Hedgehog signaling in mice. Mech. Dev. 122:223-230.

22. Romer, J.T., et al. 2004. Suppression of the Shh pathway using a small molecule inhibitor eliminates medulloblastoma in Ptc1(+/-)p53(-/-) mice. Cancer Cell. 6:229-240.

23. Berman, D.M., et al. 2002. Medulloblastoma growth inhibition by hedgehog pathway blockade. Science. 297:1559-1561.

24. Wunder, J.S., Nielsen, T.O., Maki, R.G., O’Sullivan, B., and Alman, B.A. 2007. Opportunities for improving the therapeutic ratio for patients with sarcoma. Lancet Oncol. 8:513-524.

25. Feldmann, G., et al. 2007. Blockade of hedgehog signaling inhibits pancreatic cancer invasion and metastases: a new paradigm for combination therapy in solid cancers. Cancer Res. 67:2187-2196.

26. Soriano, P. 1999. Generalized lacZ expression with the ROSA26 Cre reporter strain. Nat. Genet. 21:70-71.

27. Ren, J., Zhang, S., Kovacs, A., Wang, Y., and Muslin, A.J. 2005. Role of p38alpha MAPK in cardiac apoptosis and remodeling after myocardial infarction. J. Mol. Cell. Cardiol. 38:617-623. 